\section{AB0412 FACTORS THAT AFFECT THE RESPONSE TO TOCILIZUMAB WITH REGARD TO WORK PRODUCTIVITY IN PATIENTS WITH RHEUMATOID ARTHRITIS: A 2-YEAR FOLLOW-UP OF THE FIRST ACT- SC STUDY}

Yoshiya Tanaka ${ }^{1}$, Hideto Kameda ${ }^{2}$, Saito Kazuyoshi ${ }^{3}$, Yuko Kaneko ${ }^{4}$, Eiichi Tanaka $^{5}$, Shinsuke Yasuda ${ }^{6}$, Naoto Tamura ${ }^{7}$, Keishi Fujio $^{8}$, Takao Fujii ${ }^{9}$, Toshihisa Kojima $^{10}$, Tatsuhiko Anzai ${ }^{11}$, Yoshihisa Fujino ${ }^{1}$, Shinya Matsuda ${ }^{1}$, Hitoshi Kohsaka ${ }^{12}{ }^{2}{ }^{1}$ University of Occupational and Environmental Health, Fukuoka, Japan; ${ }^{2}$ Toho University, Tokyo, Japan; ${ }^{3}$ Tobata General Hospital, Fukuoka, Japan; ${ }^{4}$ Keio University School of Medicine, Tokyo, Japan; ${ }^{5}$ Tokyo Women's Medical University, Tokyo, Japan; ${ }^{6}$ Hokkaido University Faculty of Medicine and Graduate School of Medicine, Hokkaido, Japan; ${ }^{7}$ Juntedo University School of Medicine, Tokyo, Japan; ${ }^{8}$ The University of Tokyo, Tokyo, Japan; ${ }^{9}$ Wakayama Medical University, Wakayama, Japan; ${ }^{10}$ Nagoya University Graduate School of Medicine, Aichi, Japan; ${ }^{11}$ EPS Corporation, Tokyo, Japan; ${ }^{12}$ Tokyo Medical and Dental University, Tokyo, Japan

Background: Rheumatoid arthritis (RA) is a major cause of work disability, sickness-related absence from work, presenteeism, and loss of productivity. The decrease in work productivity that results from persistent pain, impaired mobility and function, and reduced quality of life (QOL) has important personal, societal, and economic consequences[1]. The efficacy of tocilizumab (TCZ) as monotherapy and in combination with methotrexate for RA is well known. Thus, in this study, we expected TCZ treatment to improve work productivity on the basis of interim results of up to 52 weeks[2].

Objectives: We aimed to evaluate the long-term (104 weeks) control of RA in Japanese paid workers and house workers treated with TCZ and identify factors affecting the response (responder/non-responder) to TCZ in terms of work productivity.

Methods: The FIRST ACT-SC Study (UMIN-CTR: UMIN000012306) was a cohort study that assessed the 2-year treatment outcome of TCZ-SC (administered alone or with disease modifying anti rheumatic drugs) in biologics-naive Japanese patients with RA. Logistic regression analysis was performed to determine the factors contributing to treatment response in terms of activity impairment (Al) in the WPAl questionnaire, which is an index for the evaluation of overall daily life performance. In addition, the associations of these responses to treatment withdrawal rate and QOL were also evaluated.

Results: Of the 377 enrolled participants, 357 who were treated with TCZ-SC were included in the analysis. Al was improved from $56.2 \%$ at baseline to $22.9 \%$ at 104 weeks in the patients who received TCZ. In the single regression analysis, the factors that were statistically significant in the logistic regression analysis were job type $(p=0.045), K 6$ as a measure of psychological distress $(p=0.030)$, Health Assessment Questionnaire-Disability Index (HAQ-DI; $\mathrm{p}=0.023$ ), and EuroQol five-dimensional descriptive system (EQ-5D) score $(p=0.006)$. In the multiple logistic regression analysis, disease duration, academic background, Al, EQ-5D score, ESR, and CRP level were identified as factors that affected response. The results showed a strong association between the results of the subjective assessments such as the utility value of EQ-5D. At 104 weeks, the mean difference in $\mathrm{Al}$ between the EQ-5D scores of $<0.6$ at baseline and $\geq 0.6$ was significant $(-22.94 \%$ vs $-30.50 \% ; \quad \mathrm{p}=0.029)$. Moreover, the mean difference between the HAQ-DI of $<1.5$ at baseline and $\geq 1.5$ was significant $(-20.46 \%$ vs $-29.08 \% ; p=0.013)$. A disease duration of $<1$ year was also associated with being an $\mathrm{Al}$ responder. $\mathrm{A}$ significant difference in withdrawal rate was found between the responders and non-responders $(27.0 \%$ vs. $46.9 \% ; \mathrm{p}<0.001)$, and the 2-year quality-adjusted life-year was also significantly higher in the $\mathrm{Al}$ responders.

Conclusion: Starting TCZ-SC treatment at an early stage while taking into consideration the subjective self-assessments of the patients may contribute to improvements in labour productivity and QOL, and long-term treatment continuation.

\section{REFERENCES}

[1] Best Pract Res Clin Rheumatol. 2015;29(3):495-511.

[2] Arthritis Res Ther 2018;20:151.

Disclosure of Interests: Yoshiya Tanaka Grant/research support from: Abbvie, Astellas, Bristol-Myers Squibb, Chugai, Daiichi-Sankyo, Eisai, Mitsubishi-Tanabe, MSD, Ono, Taisho-Toyama, Takeda, Speakers bureau: Abbvie, Asahi-kasei, Astellas, Bristol-Myers Squibb, Chugai, Daiichi-Sankyo, Eli Lilly, Eisai, Glaxo-Smithkline, Janssen, Mitsubishi-Tanabe, Novartis, Pfizer Japan Inc, Sanofi, Takeda, UCB, YL Biologics, Hideto Kameda Grant/research support from: AbbVie, Asahi Kasei Pharma, Astellas, Chugai, Eisai, GlaxoSmithKlein, Mitsubishi-Tanabe, Novartis, Consultant for:
AbbVie, Eli Lilly, Novartis, Speakers bureau: AbbVie, Asahi Kasei Pharma, Bristol-Myers, Chugai, Eli Lilly, Janssen, Mitsubishi-Tanabe, Novartis, Pfize, SAITO KAZUYOSHI: None declared, Yuko Kaneko Grant/ research support from: AbbVie, Eisai, Daiichi Sankyo, Sanofi, Consultant for: Bristol-Myeres Squibb, Eli Lily, Janssen, Speakers bureau: AbbVie, Eisai, Astellas, Chugai Pharmaceutical, UCB, Pfizer, Bristol-Myeres Squibb, Janssen, Tanabe-Mitsubishi, Eiichi Tanaka Consultant for: Abbvie, Ayum Pharmaceutical, Bristol Myers Squibb, Chugai Pharmaceutical, Eisai Pharmaceutical, Nippon Kayaku, Pfizer, Takeda Pharmaceutical, and UCB Pharma., Shinsuke Yasuda Grant/research support from: Bristol Myers Squibb, MSD, Speakers bureau: Chugai Parmaceutical, Tanabe- Mitsubishi Pharmaceutical, Bristol Myers Squibb, Astellas Pharmaceutical, Naoto Tamura Grant/research support from: Astellas Pharma Inc., Asahi Kasei Pharma, AYUMI Pharmaceutical Co., Chugai Pharmaceutical Co. LTD Eisai Inc., :Takeda Pharmaceutical Company Ltd., Speakers bureau: Janssen Pharmaceutical K.K., Bristol-Myers Squibb K.K., :Mitsubishi Tanabe Pharma Co., Keishi Fujio Grant/research support from: Astellas, BMS, Daiichi-Sankyo, Mitsubishi Tanabe, Pfizer, Ayumi, Takeda, Chugai, Eisai, Taisho Toyama, UCB, Janssen, Eli Lilly, and NIPPON KAYAKU., Speak ers bureau: Astellas, BMS, Daiichi-Sankyo, Mitsubishi Tanabe, Pfizer, Ayumi, Takeda, Chugai, Eisai, Taisho Toyama, UCB, Janssen, Eli Lilly, and NIPPON KAYAKU., Takao Fujii Grant/research support from: Chugai Pharmaceutical, Speakers bureau: Chugai Pharmaceutical, Toshihisa Kojima Grant/research support from: Chugai Pharmaceutical (Investigator Initiated Study), Novartis, Nippon Kayaku, Eli Lilly, Eisai, Speakers bureau: Chugai Pharmaceutical, Takeda Pharmaceutical, Pfizer, Eli Lilly Japan, Bristol Myers Squibb, Ono Pharmaceutical, Daiichi Sankyo, Astelas, UCB, Janssen Pharmaceutical, Tanabe Mitsubishi, Tatsuhiko Anza Consultant for: Chugai Pharmaceutical, Yoshihisa Fujino: None declared, Shinya Matsuda: None declared, Hitoshi Kohsaka Consultant for: CSL Behring, Speakers bureau: Japan Blood Products Organization, Ayumi Pharmacutical. and Teijin Pharmaceutical. DOI: 10.1136/annrheumdis-2019-eular.1168

\section{AB0413 THE PROSPECTIVE ASSESSMENT OF RITUXIMAB AND TOCILIZUMAB TREATMENT EFFECT ON RF-IGM AND ACPA TITRES IN RHEUMATOID ARTHRITIS PATIENTS}

Olga Borys, Bozena Targonska-Stepniak, Magdalena Dryglewska, Maria Majdan. Medical University of Lublin, Department of Rheumatology and Connective Tissue Diseases, Lublin, Poland

Background: Rheumatoid arthritis (RA) is associated with several specific autoantibodies, which can be used as diagnostic and prognostic markers. These include rheumatoid factor IgM (RF-IgM) and anti-citrullinated protein autoantibodies (ACPA). The data reporting an impact of biological disease modifying anti-rheumatic drugs (bDMARDs) on RA serological markers are divergent, probably depending on the type of treatment

Objectives: The goal of this study was to prospectively evaluate the impact of bDMARDs [rituximab (RTX) and tocilizumab (TCZ)] on titers of RA serological markers (RF IgM, ACPA), in correlation with the disease activity assessment.

Methods: The study was conducted in the consecutive 42 patients with RA (36 women, 6 men), with the mean (SD) age 48.9 (13.8) and disease duration 9.2 (3.8) years. The treatment with RTX was administered to 30 patients and with TCZ to 12 patients. The following parameters were assessed for all patients: joint counts, disease activity index of 28 joints (DAS28), complete blood cell counts, erythrocyte sedimentation rate (ESR), serum concentration of serum amyloid A (SAA), RF-lgM, ACPA. Blood samples were taken before the drug administration (baseline) and in consecutive months ( 3,6 and 9 months).

Results: Parameters of the disease activity decreased significantly in consecutive months, in comparison with the baseline assessment, in both treatment groups. The mean (SD) DAS28 decreased significantly in both groups (RTX and TCZ): respectively at baseline: 5.75 (0.69) and 5.47 (0.51); month 3: 3.92 (1.13) and 2.84 (0.76); month 6: 3.23 (1.09) and 2.3 (0.94); month 9: 3.67 (1.19) and $2.12(0.81)$. The mean (SD) SAA concentration was significantly lower in consecutive months, in both groups (RTX and TCZ): respectively at baseline: 408.5 (268.2) and 402.0 (291.0) $\mathrm{ug} / \mathrm{ml}$; month 3: 214.2 (255.3) and 83.6 (157.8) ug/ml; month 6: 100.2 (154.9) and $31.5(46.3) \mathrm{ug} / \mathrm{ml}$; month 9: 108.5 (128.8) and 26.7 (32.4) $\mathrm{ug} / \mathrm{ml}$. The significant reduction of RA specific autoantibodies (RFIgM and ACPA) was noted in the group of patients treated with RTX (Figure 1, Figure 2). Treatment with TCZ did not affect significantly RA specific autoantibodies up to 6 months of therapy; the significant decrease of ACPA concentration was noted only between baseline and 
month 9 (Figure 3); no significant change of RF-IgM was observed (Figure 4).

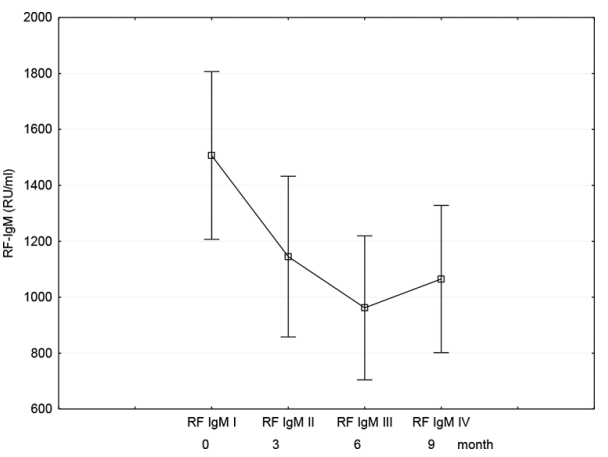

Figure 1. RF-IgM concentrations during RTX treatment.

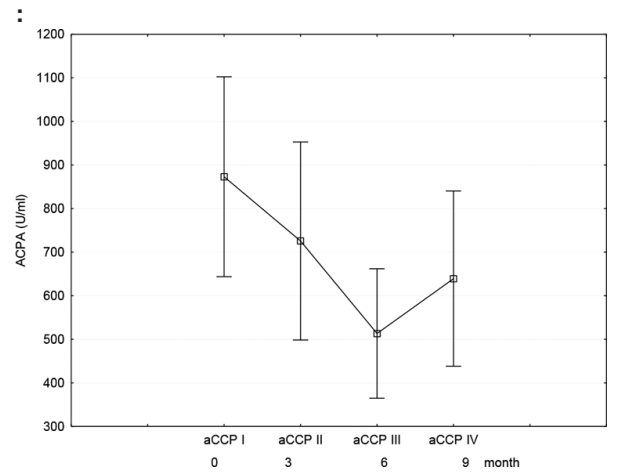

Figure 2. ACPA concentrations during RTX treatment.

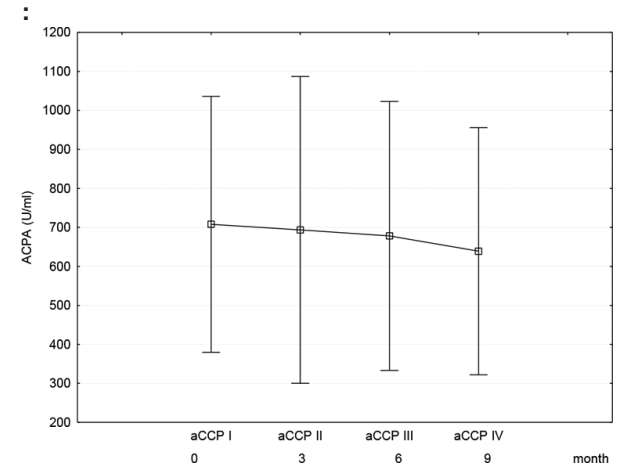

Figure 3. ACPA concentrations during TCZ treatment.

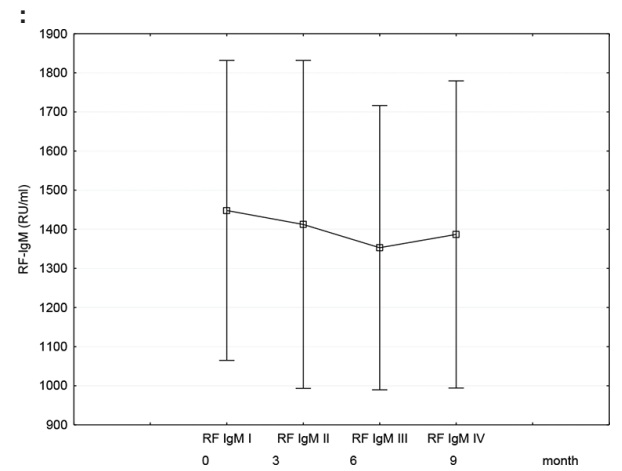

Figure 4. RF-IgM concentrations during TCZ treatment.
Conclusion: The results of the study suggest that both bDMARDs reduced significantly the disease activity in RA patients. The treatment with both drugs was associated with a significant reduction of SAA. However only RTX treatment affected significantly the production of disease specific autoantibodies. Long-term observation is necessary to assess a reliable effect of bDMARDs on the production of marker autoantibodies in association with the disease activity. Disclosure of Interests: Olga Borys: None declared, Bozena Targonska-Stepniak Speakers bureau: Sandoz, Berlin-Chemie, Magdalena Dryglewska: None declared, Maria Majdan Speakers bureau: MSD, UCB, Abbvie, Roche DOI: 10.1136/annrheumdis2019-eular.3476

\section{AB0414 THE IN VITRO EFFECT OF BIOLOGICAL AND CONVENTIONAL DISEASE MODIFYING ANTI RHEUMATIC DRUGS (DMARDS) ON FIBROCYTE DIFFERENTIATION IN RHEUMATOID ARTHRITIS PATIENTS AND HEALTHY CONTROLS}

Helene Tenstad ${ }^{1}$, Pernille Just Vinholt ${ }^{2}$, Christian Nielsen ${ }^{3}$, Hanne Merete Lindegaard ${ }^{1}$, Søren Andreas Just ${ }^{4}{ }^{1}$ Odense University Hospital, Department of Rheumatology, Odense, Denmark; ${ }^{2}$ Odense University Hospital, Department of Biochemistry and Pharmacology, Odense, Denmark; ${ }^{3}$ Odense University Hospital, Department of Immunology, Odense, Denmark; ${ }^{4}$ Odense University Hospital - Svendborg, Department of internal medicin - Section of Rheumatology, Svendborg, Denmark

Background: Fibrocytes are circulating cells with both myeloid and haemopoietic properties. They home in tissues with active inflammation, where they differentiate into mature fibrocytes that are involved in several inflammatory pathways. One complication of Rheumatoid Arthritis (RA) is Interstitial Lung Disease (ILD) resulting in high mortality and with limited treatment options. Fibrocyte levels are elevated in RA patients compared with healthy individuals and further increased in RA patients with signs of ILD (reduced diffusion capacity) and in patients with idiopathic lung fibrosis (IPF) or scleroderma. Thus, fibrocytes have been proposed as a future treatment target.

Objectives: We investigate the effect of corticosteroids, conventional Disease Modifying Anti Rheumatic Drugs (cDMARDs) and biological DMARDs (bDMARDs) on the in vitro differentiation of isolated peripheral blood mononuclear cells (PBMCs) into mature fibrocytes.

Methods: 10 participants were included (five patients with RA and five healthy controls). Information on current medication, sex, age, serology and disease activity were collected. PBMCS were isolated and cultured for 5 days in four wells per drug. Drugs included prednisolone, CDMARD (Methotrexate, Sulfasalasine, Hydroxycloroquine) and bDMARD (Inflectra, Etanecept, Tocilizumab, Adalimumab, Abatacept, Rituximab), and control wells with no drugs.

Results: Overall, abatacept and prednisolone significantly suppressed differentiation of PBMC into fibrocytes compared to control wells, see Figure $1(p=0.02$ and $p<0.01$, respectively) $(n=10)$. The reductive effect of Abatacept was significant among RA patients ( $p=0.009$ and) but not among healthy subjects. In overall analysis $(n=10)$, Abatacept reduced fibrocyte levels with an average of $44 \%$ overall and in the RA group $71 \%$ compared to control wells. Tocilizumab reduced the fibrocyte count with $63 \%$ overall and $45 \%$ in the RA group, although not significant $(p=0.07$ and $\mathrm{p}=0.06$ respectively)

Conclusion: Abatacept and prednisolon suppress the differentiation of mononuclear cells to mature fibrocytes in vitro in RA patients and data indicating a similar effect of Tocilizumab. Prednisolone are used in the treatment of RA-ILD but has a marked toxicity, so new treatment modalities are desirable. Our findings are in line with the fact that fibrocytes have the receptors targeted by abatacept, furthermore recent scleroderma research has shown Abatacept to reduce fibrocyte levels in vitro and Tocilizumab to potentially reduce lung and skin affection $(1,2)$ Further research using abatacept and tocilizumab to target fibrocytes are needed in order investigate the treatment potential of these drugs in RA-ILD. 